\title{
Surface-Enhanced Spectroscopy for Higher-Order Light Scattering: A Combined Experimental and Theoretical Study of Second Hyper-Raman Scattering
}

Philip D. Simmons, Jr., ${ }^{\dagger}$ Hubert K Turley, ${ }^{\S}$ Daniel W. Silverstein, ${ }_{\S}$ Lasse Jensen, ${ }^{*}, \|$ Jon. P. Camden ${ }^{*}$,

'Department of Chemistry, University of Tennessee, Knoxville, Tennessee 37996, United States

${ }^{\S}$ Department of Chemistry and Biochemistry, University of Notre Dame, Notre Dame, Indiana

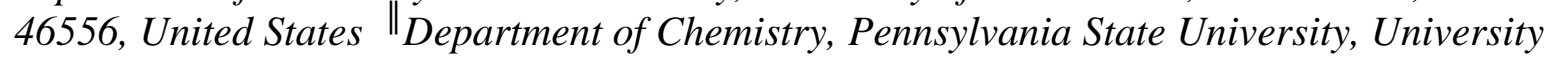
Park, Pennsylvania 16802, United States

\section{Resonant Second Hyperpolarizability}

Simulations of resonance second hyper-Raman (R2HR) scattering require the calculation of the resonant second hyperpolarizability. Based on the expression for the second hyperpolarizability $(\gamma),{ }^{1}$ when three degenerate photons with frequency $\omega_{L}$ are absorbed by the molecule, $\gamma$ is written:

$$
\begin{aligned}
\gamma_{\rho \sigma \tau v}\left(-3 \omega_{L} ; \omega_{L}, \omega_{L}, \omega_{L}\right) & \sum_{J, j, K, k, L, l}\left(\frac{\left\langle F_{0}\left|\mu_{\rho}^{0 l}\right| L_{l}\right\rangle\left\langle L_{l}\left|\mu_{v}^{l k}\right| K_{k}\right\rangle\left\langle K_{k}\left|\mu_{\tau}^{k j}\right| J_{j}\right\rangle\left\langle J_{j}\left|\mu_{\sigma}^{j 0}\right| I_{0}\right\rangle}{\left(E_{J_{j}}-E_{I_{0}}-\omega_{L}\right)\left(E_{K_{k}}-E_{I_{0}}-2 \omega_{L}\right)\left(E_{L_{l}}-E_{I_{0}}-3 \omega_{L}-i \Gamma_{l}\right)}\right. \\
& +\frac{\left\langle F_{0}\left|\mu_{\rho}^{0 l}\right| L_{l}\right\rangle\left\langle L_{l}\left|\mu_{\tau}^{l k}\right| K_{k}\right\rangle\left\langle K_{k}\left|\mu_{v}^{k j}\right| J_{j}\right\rangle\left\langle J_{j}\left|\mu_{\sigma}^{j 0}\right| I_{0}\right\rangle}{\left(E_{J_{j}}-E_{I_{0}}-\omega_{L}\right)\left(E_{K_{k}}-E_{I_{0}}-2 \omega_{L}\right)\left(E_{L_{l}}-E_{I_{0}}-3 \omega_{L}-i \Gamma_{l}\right)} \\
& +\frac{\left\langle F_{0}\left|\mu_{\rho}^{0 l}\right| L_{l}\right\rangle\left\langle L_{l}\left|\mu_{v}^{l k}\right| K_{k}\right\rangle\left\langle K_{k}\left|\mu_{\sigma}^{k j}\right| J_{j}\right\rangle\left\langle J_{j}\left|\mu_{\tau}^{j 0}\right| I_{0}\right\rangle}{\left(E_{J_{j}}-E_{I_{0}}-\omega_{L}\right)\left(E_{K_{k}}-E_{I_{0}}-2 \omega_{L}\right)\left(E_{L_{l}}-E_{I_{0}}-3 \omega_{L}-i \Gamma_{l}\right)} \\
& +\frac{\left\langle F_{0}\left|\mu_{\rho}^{0 l}\right| L_{l}\right\rangle\left\langle L_{l}\left|\mu_{\tau}^{l k}\right| K_{k}\right\rangle\left\langle K_{k}\left|\mu_{\sigma}^{k j}\right| J_{j}\right\rangle\left\langle J_{j}\left|\mu_{v}^{j 0}\right| I_{0}\right\rangle}{\left(E_{J_{j}}-E_{I_{0}}-\omega_{L}\right)\left(E_{K_{k}}-E_{I_{0}}-2 \omega_{L}\right)\left(E_{L_{l}}-E_{I_{0}}-3 \omega_{L}-i \Gamma_{l}\right)} \\
& +\frac{\left\langle F_{0}\left|\mu_{\rho}^{0 l}\right| L_{l}\right\rangle\left\langle L_{l}\left|\mu_{\sigma}^{l k}\right| K_{k}\right\rangle\left\langle K_{k}\left|\mu_{v}^{k j}\right| J_{j}\right\rangle\left\langle J_{j}\left|\mu_{\tau}^{j 0}\right| I_{0}\right\rangle}{\left(E_{J_{j}}-E_{I_{0}}-\omega_{L}\right)\left(E_{K_{k}}-E_{I_{0}}-2 \omega_{L}\right)\left(E_{L_{l}}-E_{I_{0}}-3 \omega_{L}-i \Gamma_{l}\right)} \\
& \left.+\frac{\left\langle F_{0}\left|\mu_{\rho}^{0 l}\right| L_{l}\right\rangle\left\langle L_{l}\left|\mu_{\sigma}^{l k}\right| K_{k}\right\rangle\left\langle K_{k}\left|\mu_{\tau}^{k j}\right| J_{j}\right\rangle\left\langle J_{j}\left|\mu_{v}^{j 0}\right| I_{0}\right\rangle}{\left(E_{J_{j}}-E_{I_{0}}-\omega_{L}\right)\left(E_{K_{k}}-E_{I_{0}}-2 \omega_{L}\right)\left(E_{L_{l}}-E_{I_{0}}-3 \omega_{L}-i \Gamma_{l}\right)}\right)
\end{aligned}
$$

Here, we assume the Born-Oppenheimer approximation. The ground electronic state is written as $|0\rangle$, while the intermediate electronic states are $|j\rangle,|k\rangle$, and $|l\rangle$. The initial and final vibrational states are $\left|I_{0}\right\rangle$ and $\left|F_{0}\right\rangle$, while intermediate vibrational states are $\left|J_{j}\right\rangle,\left|K_{k}\right\rangle$, and $\left|L_{l}\right\rangle$. We employ the shorthand notation $\mu_{\sigma}^{j 0}=\left\langle j\left|\mu_{\sigma}\right| 0\right\rangle$ for the transition dipole moment. Assuming 
no intermediate resonance exists with a one- or two-photon transition, we may perform a closure over intermediate states $\left|J_{j}\right\rangle$ and $\left|K_{k}\right\rangle$, which results in the following expression:

$$
\begin{aligned}
\gamma_{\rho \sigma \tau \nu}\left(-3 \omega_{L} ;\right. & \left.\omega_{L}, \omega_{L}, \omega_{L}\right) \\
& =\sum_{j, k, L, l} \frac{\left\langle F_{0}\left|\mu_{\rho}^{0 l}\right| L_{l}\right\rangle}{\left(E_{L_{l}}-E_{I_{0}}-3 \omega_{L}-i \Gamma_{l}\right)}\left\langle L_{l}\right|\left(\frac{\mu_{v}^{l k} \mu_{\tau}^{k j} \mu_{\sigma}^{j 0}}{\left(E_{j}-E_{0}-\omega_{L}\right)\left(E_{k}-E_{0}-2 \omega_{L}\right)}\right. \\
& +\frac{\mu_{\tau}^{l k} \mu_{v}^{k j} \mu_{\sigma}^{j 0}}{\left(E_{j}-E_{I_{0}}-\omega_{L}\right)\left(E_{k}-E_{0}-2 \omega_{L}\right)}+\frac{\mu_{v}^{l k} \mu_{\sigma}^{k j} \mu_{\tau}^{j 0}}{\left(E_{j}-E_{0}-\omega_{L}\right)\left(E_{k}-E_{0}-2 \omega_{L}\right)} \\
& +\frac{\mu_{\tau}^{l k} \mu_{\sigma}^{k j} \mu_{v}^{j 0}}{\left(E_{j}-E_{I_{0}}-\omega_{L}\right)\left(E_{k}-E_{0}-2 \omega_{L}\right)}+\frac{\mu_{v}^{k j} \mu_{\tau}^{j 0}}{\left(E_{j}-E_{0}-\omega_{L}\right)\left(E_{k}-E_{0}-2 \omega_{L}\right)} \\
& \left.+\frac{\mu_{\sigma}^{l k} \mu_{\tau}^{k j} \mu_{v}^{j 0}}{\left(E_{j}-E_{I_{0}}-\omega_{L}\right)\left(E_{k}-E_{0}-2 \omega_{L}\right)}\right)\left|I_{0}\right\rangle
\end{aligned}
$$

Or, alternatively:

$$
\begin{aligned}
& \gamma_{\rho \sigma \tau v}\left(-3 \omega_{L} ; \omega_{L}, \omega_{L}, \omega_{L}\right) \\
& =\sum_{L, l} \frac{\left\langle F_{0}\left|\mu_{\rho}^{0 l}\right| L_{l}\right\rangle}{\left(E_{L_{l}}-E_{I_{0}}-3 \omega_{L}-i \Gamma_{l}\right)}\left\langle L_{l}\left|\left(\sum \mathcal{P}_{\sigma \tau v} \sum_{j, k} \frac{\mu_{\sigma}^{l k} \mu_{\tau}^{k j} \mu_{v}^{j 0}}{\left(E_{j}-E_{0}-\omega_{L}\right)\left(E_{k}-E_{0}-2 \omega_{L}\right)}\right)\right| I_{0}\right\rangle \\
& =\sum_{L, l} \frac{\left\langle F_{0}\left|\mu_{\rho}^{0 l}\right| L_{l}\right\rangle\left\langle L_{l}\left|T_{\sigma \tau \nu}^{l 0}\right| I_{0}\right\rangle}{\left(E_{L_{l}}-E_{I_{0}}-3 \omega_{L}-i \Gamma_{l}\right)}
\end{aligned}
$$

Inspection of the expression for $\gamma$ allows it to be simplified. After performing the closure over states $\left|J_{j}\right\rangle$ and $\left|K_{k}\right\rangle$, we have a summation of six terms. Each term is a permutation of the indices $\sigma, \tau$, and $v$. This allows the six terms to be written using the permutation operator $\sum \mathcal{P}_{\sigma \tau \nu}$. This expression is then noticed to be identical to the three-photon transition tensor, $T_{\sigma \tau \nu}^{l 0}{ }^{2}$

From this step, it is possible to write the expression for $\gamma$ using the time-dependent formalism, using a procedure identical to that shown in previous work. ${ }^{3}$ Assuming the Franck-Condon approximation, the resulting expression for the resonant second hyperpolarizability becomes

$$
\gamma_{\rho \sigma \tau \nu}\left(-3 \omega_{L} ; \omega_{L}, \omega_{L}, \omega_{L}\right)=\sum_{l}\left(\mu_{\rho}^{0 l}\right)^{e q}\left(T_{\sigma \tau \nu}^{l 0}\right)^{e q} i \int_{0}^{\infty} d t\left\langle F_{0} \mid I_{l}(t)\right\rangle e^{i\left(\epsilon_{I_{0}}+3 \omega_{L}\right) t-\Gamma_{l} t}
$$

The notation $(x)^{e q}$ indicates a transition moment evaluated at the ground state equilibrium position. A Gaussian wavepacket $\left|I_{l}(t)\right\rangle$ is propagated on excited state $|l\rangle$. 


\section{Differential Second Hyper-Raman Scattering Cross Section}

The intensity of R2HR scattering is related to the differential second hyper-Raman scattering cross section with the following relationship:

$$
I^{R 2 H R}=\left(\frac{d \sigma^{R S H R}}{d \Omega}\right) I_{0}^{3},
$$

where $I_{0}$ is the irradiance. Assuming the scattered radiation is collected along the experimental $\mathrm{X}$-axis and the incident radiation is polarized along the experimental Y-axis, the differential second hyper-Raman scattering cross section is written:

$$
\frac{d \sigma^{R 2 H R}}{d \Omega}=\frac{64 \pi^{2} \alpha^{4} h^{4}}{e^{8} c^{2}} \omega_{s}^{4}\left[\left\langle\gamma_{Y Y Y Y}^{2}\right\rangle+\left\langle\gamma_{Z Y Y Y}^{2}\right\rangle\right]
$$

where $\alpha$ is the fine structure constant, $h$ is Planck's constant, $e$ is the fundamental charge, $c$ is the speed of light, and $\omega_{s}=3 \omega_{L}-\omega_{\mathrm{v}}$ is the scattered frequency for the normal mode with frequency $\omega_{\mathrm{v}}$.

The orientational averages for the differential cross section are non-trivial to evaluate by hand and prone to mathematical errors. Instead, the approach adopted by Andrews et al. ${ }^{4-5}$ was implemented in Python to automate the procedure of evaluating the orientational averages.

For the expressions of the orientational averages, it is assumed that tensor element $\gamma_{a b c d} \propto$ $\mu_{a} T_{b c d}$. This allows simplifications of the orientational averages due to the symmetry of the three-photon transition tensor. For example, we have that $\gamma_{x x x z}=\gamma_{x x z x}=\gamma_{x z x x} \neq \gamma_{z x x x}$, both because $\mu_{x}$ is not necessarily equal to $\mu_{z}$, and because $T_{x x z}=T_{x z x}=T_{z x x}$ but these are not necessarily equal to $T_{x x x}$. Expressions for the required orientational averages are given below.

$$
\begin{gathered}
\left\langle\gamma_{Y Y Y Y}^{2}\right\rangle=G_{1}^{Y Y Y Y}+G_{2}^{Y Y Y Y}+G_{3}^{Y Y Y Y}+G_{4}^{Y Y Y Y} \\
G_{1}^{Y Y Y Y}=\frac{1}{9} \sum_{a} \gamma_{a a a a}^{2} \\
G_{2}^{Y Y Y Y}=\frac{1}{7} \sum_{a \neq b} \gamma_{a a a b}^{2}+\frac{2}{21} \sum_{a \neq b} \gamma_{a a a a} \gamma_{a a b b}+\frac{2}{21} \sum_{a \neq b} \gamma_{a a a b} \gamma_{b a a a}+\frac{1}{63} \sum_{a \neq b} \gamma_{b a a a}^{2} \\
+\frac{2}{21} \sum_{a \neq b} \gamma_{a a a a} \gamma_{b a a b} \\
G_{3}^{Y Y Y Y}=\frac{3}{35} \sum_{a \neq b} \gamma_{a a b b}^{2}+\frac{2}{35} \sum_{a \neq b} \gamma_{a a a b} \gamma_{a b b b}+\frac{2}{105} \sum_{a \neq b} \gamma_{a b b b} \gamma_{b a a a}+\frac{6}{35} \sum_{a \neq b} \gamma_{a a b b} \gamma_{b a a b} \\
+\frac{3}{35} \sum_{a \neq b} \gamma_{b a a b}^{2}+\frac{2}{35} \sum_{a \neq b} \gamma_{b a a a} \gamma_{b a b b}+\frac{6}{35} \sum_{a \neq b} \gamma_{a a a b} \gamma_{b b b a}+\frac{2}{105} \sum_{a \neq b} \gamma_{a a a a} \gamma_{b b b b}
\end{gathered}
$$




$$
\begin{aligned}
& G_{4}^{Y Y Y Y}=\frac{2}{35} \sum_{a \neq b \neq c} \gamma_{a a b c}^{2}+\frac{1}{35} \sum_{a \neq b \neq c} \gamma_{a a b b} \gamma_{a a c c}+\frac{2}{35} \sum_{a \neq b \neq c} \gamma_{a a a c} \gamma_{a b b c}+\frac{2}{105} \sum_{a \neq b \neq c} \gamma_{a b c c} \gamma_{b a a a} \\
& +\frac{2}{35} \sum_{a \neq b \neq c} \gamma_{a a c c} \gamma_{b a a b}+\frac{4}{35} \sum_{a \neq b \neq c} \gamma_{a a b c} \gamma_{b a a c}+\frac{1}{105} \sum_{a \neq b \neq c} \gamma_{b a a c}^{2} \\
& +\frac{4}{35} \sum_{a \neq b \neq c} \gamma_{a a a c} \gamma_{b a b c}+\frac{2}{35} \sum_{a \neq b \neq c}^{a \neq c \neq c} \gamma_{a a a b} \gamma_{b a c c}+\frac{2}{105} \sum_{a \neq b \neq c}^{a \neq b \neq c} \gamma_{b a a a} \gamma_{b a c c} \\
& +\frac{1}{63} \sum_{a \neq b \neq c}^{a \neq b \neq c} \gamma_{a a a a} \gamma_{b b c c}+\frac{4}{105} \sum_{a \neq b \neq c}^{a \neq b \neq c} \gamma_{b a b c} \gamma_{c a a a}+\frac{1}{35} \sum_{a \neq b \neq c}^{a \neq b \neq c} \gamma_{b a a c} \gamma_{c a a b} \\
& +\frac{1}{35} \sum_{a \neq b \neq c} \gamma_{b a a b} \gamma_{c a a c}+\frac{1}{315} \sum_{a \neq b \neq c}^{a \neq b \neq c} \gamma_{a a a a} \gamma_{c b b c} \\
& \left\langle\gamma_{Z Y Y Y}^{2}\right\rangle=G_{1}^{Z Y Y Y}+G_{2}^{Z Y Y Y}+G_{3}^{Z Y Y Y}+G_{4}^{Z Y Y Y} \\
& G_{1}^{Z Y Y Y}=\frac{1}{63} \sum_{a} \gamma_{a a a a}^{2} \\
& G_{2}^{Z Y Y Y}=\frac{2}{35} \sum_{a \neq b} \gamma_{a a a b}^{2}+\frac{4}{105} \sum_{a \neq b} \gamma_{a a a a} \gamma_{a a b b}-\frac{1}{21} \sum_{a \neq b} \gamma_{a a a b} \gamma_{b a a a}+\frac{4}{63} \sum_{a \neq b} \gamma_{b a a a}^{2} \\
& -\frac{1}{21} \sum_{a \neq b} \gamma_{a a a a} \gamma_{b a a b} \\
& G_{3}^{Z Y Y Y}=\frac{3}{35} \sum_{a \neq b} \gamma_{a a b b}^{2}+\frac{2}{35} \sum_{a \neq b} \gamma_{a a a b} \gamma_{a b b b}-\frac{1}{105} \sum_{a \neq b} \gamma_{a b b b} \gamma_{b a a a}-\frac{3}{35} \sum_{a \neq b} \gamma_{a a b b} \gamma_{b a a b} \\
& +\frac{3}{35} \sum_{a \neq b} \gamma_{b a a b}^{2}-\frac{3}{35} \sum_{a \neq b} \gamma_{a a a b} \gamma_{b b b a}+\frac{2}{35} \sum_{a \neq b} \gamma_{b a a a} \gamma_{b b b a}-\frac{1}{105} \sum_{a \neq b} \gamma_{a a a a} \gamma_{b b b b} \\
& G_{4}^{Z Y Y Y}=\frac{2}{35} \sum_{a \neq b \neq c} \gamma_{a a b c}^{2}+\frac{1}{35} \sum_{a \neq b \neq c} \gamma_{a a b b} \gamma_{a a c c}+\frac{2}{35} \sum_{a \neq b \neq c} \gamma_{a a a c} \gamma_{a b b c}-\frac{1}{105} \sum_{a \neq b \neq c} \gamma_{a b c c} \gamma_{b a a a} \\
& -\frac{1}{35} \sum_{a \neq b \neq c} \gamma_{a a c c} \gamma_{b a a b}-\frac{2}{35} \sum_{a \neq b \neq c} \gamma_{a a b c} \gamma_{b a a c}+\frac{4}{35} \sum_{a \neq b \neq c} \gamma_{b a a c}^{2} \\
& -\frac{2}{35} \sum_{a \neq b \neq c}^{a \neq b \neq c} \gamma_{a a a c} \gamma_{b a b c}-\frac{1}{35} \sum_{a \neq b \neq c}^{a \neq b \neq c} \gamma_{a a a b} \gamma_{b a c c}+\frac{8}{105} \sum_{a \neq b \neq c}^{a \neq b \neq c} \gamma_{b a a a} \gamma_{b a c c} \\
& -\frac{1}{105} \sum_{a \neq b \neq c} \gamma_{a a a a} \gamma_{b b c c}-\frac{1}{210} \sum_{a \neq b \neq c} \gamma_{b a b c} \gamma_{c a a a}-\frac{1}{70} \sum_{a \neq b \neq c} \gamma_{b a a c} \gamma_{c a a b} \\
& -\frac{1}{70} \sum_{a \neq b \neq c}^{a \neq b \neq c} \gamma_{b a a b} \gamma_{c a a c}-\frac{1}{70} \sum_{a \neq b \neq c}^{a \neq b \neq c} \gamma_{b a a a} \gamma_{c a b c}
\end{aligned}
$$




\section{Three-Photon Absorption Cross Sections}

The three-photon absorption (3PA) cross section is given by

$$
\sigma_{3 P A}^{F C}(\omega)=8 \pi^{4} \alpha^{3} \omega^{3} \sum_{k} \delta_{3 P A}^{0 k} R e \int_{0}^{\infty} d t\left\langle I_{0} \mid I_{k}(t)\right\rangle e^{i\left(\epsilon_{I_{0}}+3 \omega\right) t-\Gamma_{k} t}
$$

where the three-photon transition probability for state $\mathrm{k}$ is given by ${ }^{6}$

$$
\delta_{3 P A}^{0 k}=\frac{1}{35}\left(3 \sum_{a b c} T_{a a b}^{0 k} T_{c c b}^{0 k}+3 \sum_{a b c} T_{a b c}^{0 k} T_{a b c}^{0 k}\right)
$$

in terms of the three-photon transition tensor.

\section{Enhancement Factor Spectra}

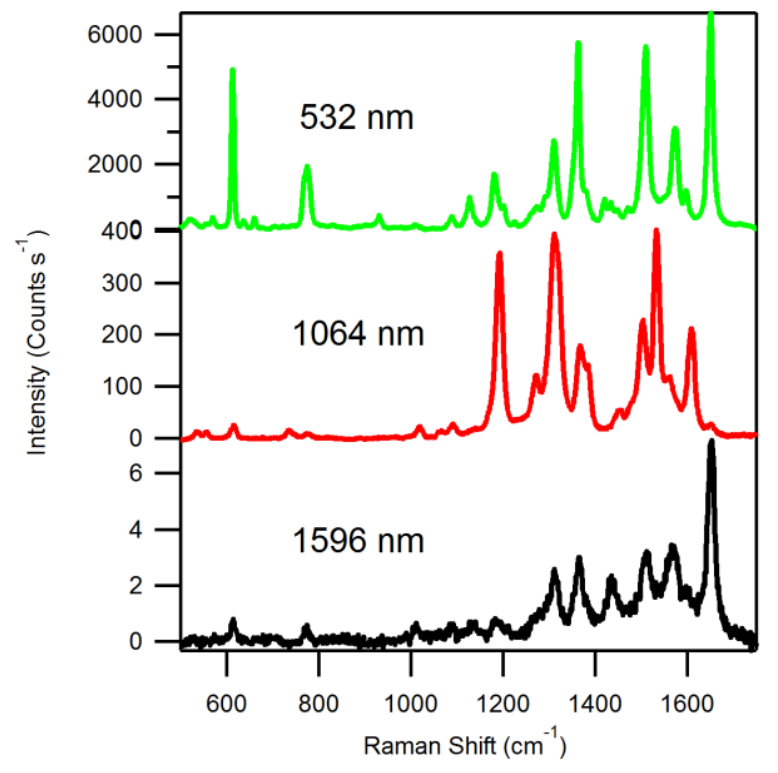

Figure 1. SERS (green), SEHRS (red), and SE2HRS (black) of R6G $\left(10^{-7} \mathrm{M}\right)$ obtained using 532 $\mathrm{nm}, 1064 \mathrm{~nm}$, and $1596 \mathrm{~nm}$ excitations, respectively. The SERS was taken for $10 \mathrm{~s}$ at $30 \mu \mathrm{W}$, the SEHRS was taken for $30 \mathrm{~s}$ at $2 \mathrm{~mW}$, and the SE2HRS was taken for $4 \mathrm{~min}$ at $2 \mathrm{~mW}$. The spectra were baseline corrected and integrated over all peaks using a peak fitting routine in Igor software to determine the experimental surface-enhanced response. 


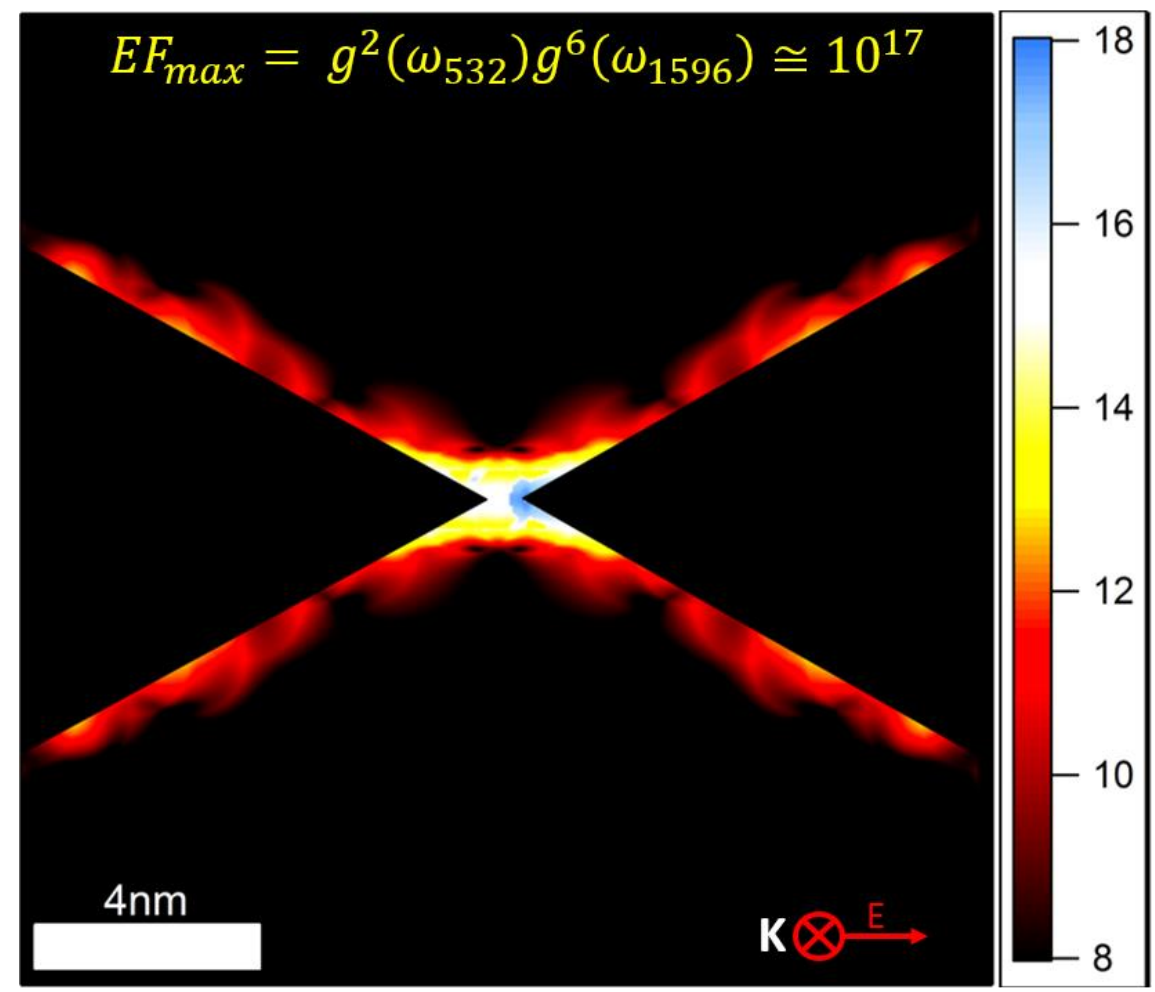

Figure 2. The plasmon map of a silver bowtie dimer (equilateral triangle, side length $50 \mathrm{~nm}$, $25 \mathrm{~nm}$ thickness, $1 \mathrm{~nm}$ gap) was computed via the discrete dipole approximation (DDA) ${ }^{7}$ with incident frequencies $\left(\omega_{532}, \omega_{1596}\right)$ to give the approximate local field enhancement relative to the driving field over the entire substrate. The plasmon map is plotted at a depth of $11 \mathrm{~nm}$ and is zoomed in to show area of high field intensity. The calculations yielded an $\mathrm{EF}_{\max } \sim 10^{17}$ arising from inside the nanoparticle junction and an $\mathrm{EF}_{\text {average }} \sim 10^{13}$ from over the entire substrate.

\section{$\underline{\text { References }}$}

(1) Christie, J. H.; Lockwood, D. J., Selection Rules for Three- and Four-Photon Raman Interactions. J. Chem. Phys. 1971, 54, 1141-1154.

(2) Cronstrand, P.; Luo, Y.; Norman, P.; Ågren, H., Ab Initio Calculations of Three-Photon Absorption. Chem. Phys. Lett. 2003, 375, 233-239.

(3) Silverstein, D. W.; Jensen, L., Vibronic Coupling Simulations for Linear and Nonlinear Optical Processes: Theory. J. Chem. Phys. 2012, 136, 064111.

(4) Andrews, D. L.; Thirunamachandran, T., On Three-Dimensional Rotational Averages. J. Chem. Phys. 1977, 67, 5026-5033.

(5) Andrews, D. L.; Ghoul, W. A., Eighth Rank Isotropic Tensors and Rotational Averages. J. Phys. A-Math. Gen. 1981, 14, 1281.

(6) Cronstrand, P.; Jansik, B.; Jonsson, D.; Luo, Y.; Agren, H., Density Functional Response Theory Calculations of Three-Photon Absorption. J. Chem. Phys. 2004, 121, 9239-9246. 
(7) Draine, B. T.; Flatau, P. J., Discrete-Dipole Approximation for Scattering Calculations. JOSA A 1994, 11, 1491-1499. 